\title{
On the use of the Internet to collect earthquake information
}

\section{TABLE OF CONTENTS}

On the use of the Internet to collect earthquake information

Rémy Bossu and Paul S. Earle

Using Internet reports for early estimates of the final death toll of earthquake-generated tsunami: the March 11, 2011, Tohoku, Japan, earthquake

Xiaolin Yang, Zhongliang Wu, Yingchun Li

Crowdsourcing earthquake damage assessment using remote sensing imagery

Luke Barrington, Shubharoop Ghosh, Marjorie Greene, Shay Har-Noy, Jay Berger, Stuart Gill, Albert Yu-Min Lin, Charles Huyck

USGS “Did You Feel It?” Internet-based macroseismic intensity maps

David J. Wald, Vincent Quitoriano, Bruce Worden, Margaret Hopper, James W. Dewey

Twitter earthquake detection: earthquake monitoring in a social world

Paul S. Earle, Daniel C. Bowden, Michelle Guy

Flash sourcing, or rapid detection and characterization of earthquake effects through website traffic analysis

Rémy Bossu, Sébastien Gilles, Gilles Mazet-Roux, Fréderic Roussel, Laurent Frobert, Linus Kamb

Comparison between low-cost and traditional MEMS accelerometers: a case study from the M7.1 Darfield, New Zealand, aftershock deployment

Elizabeth S. Cochran, Jesse F. Lawrence, Anna Kaiser, Bill Fry, Angela Chung, Carl Christensen

Community Seismic Network

Robert W. Clayton, Thomas Heaton, Mani Chandy, Andreas Krause, Monica Kohler, Julian Bunn, Richard Guy, Michael Olson,

Mathew Faulkner, MingHei Cheng, Leif Strand, Rishi Chandy, Daniel Obenshain, Annie Liu, Michael Aivazis 


\section{ON THE USE OF THE INTERNET TO COLLECT EARTHQUAKE INFORMATION}

As geophysicists, we find it difficult to imagine our daily professional and personal lives without the Internet. Emails have been the mainstay of scientific communication since the 1980's and websites, since their inception in the 1990's, have grown to represent the heart of public information dissemination. Now, scientific data flow in real-time around the world through custom protocols, portals, and web services, and the advent of social media services such as Facebook and Twitter offer both a new mechanism to communicate ideas and a potential resource for collecting data.

In this issue, authors illustrate ways to augment our traditional methods of collecting data with information obtained through the Internet. Some of these studies, including the public interpretation of remote sensing data (Barrington et al.), are still in their early stages, while others, such as, "Did You Feel It?" (Wald et al.), were in existence before the term "crowd-sourcing" was even coined. The seven papers in this issue can be divided into two different categories: studies that actively engage the public to gather or process data, and those that obtain geophysical insight from Internet-based information that is originally intended for other purposes.

The first category is related to crowd-sourcing initiatives. Crowd-sourcing refers to work being done or data being contributed by an unidentified "crowd" of people focusing on solving a specific task. The GEO-CAN initiative (Barrington et al.) uses crowd-sourced analysis of remote sensing imagery to speed the assessment of damage caused by natural disasters. Two papers discuss expanding the observational base of seismic ground motions by crowd sourcing citizenoperated seismic networks. Cochran et al. show that the Quake Catcher Network (QCN) can be quickly and inexpensively deployed for aftershock surveys and for the collection of strong-motion data. The Community Seismic Network (CSN) intends to imbed a dense array of low-cost sensors within a traditional seismic network to rapidly produce maps of ground shaking, potentially before accurate estimates of location and magnitude are obtained (Clayton et al.). Wald et al. present a retrospective of "Did You Feel It?", one of the first Internet-based scientific crowd-sourcing initiatives.

The second type of study gathers Internet-based data not originally intended for geophysical analysis. Two studies analyse the online reaction of eyewitnesses in the immediate aftermath of an earthquake's occurrence to detect felt earthquakes and to characterise their impact within minutes of the earthquake's occurrence. Bossu et al. analyse fluctuations in the use of the European-Mediterranean Seismological Centre's (EMSC) real time earthquake information website and explain how such information can potentially detect damage and be used to improve public information regarding recent events. Earle et al. show how Twitter, the real-time micro blogging site, can be used to detect felt earthquakes independent of seismic monitoring systems. Another Internet-based study by Yang et al. collect Internet reports of the March 11, 2011 Tohoku, Japan, earthquake to show that they could have provided useful early estimates of the final death toll.

These studies are based on citizen engagement and they modify the ways in which we, as a scientific community, interface with society at large. In addition to the data they supply, these Internet-based techniques require geophysicists to better understand and respond to public demands and expectations after natural disasters. They will continue to transform the services we provide to society on a daily basis.

Rémy Bossu

European Mediterranean Seismological Centre (EMSC),

earthquake information website (www.emsc-csem.org)
Paul S. Earle

U.S. Geological Survey, Denver, CO, USA 